\title{
Plasmonic contribution to the van der Waals energy in strongly interacting bilayers
}

\author{
Murat Tas and B. Tanatar \\ Department of Physics, Bilkent University, Bilkent, Ankara 06800, Turkey \\ (Received 12 May 2009; revised manuscript received 24 February 2010; published 23 March 2010)
}

\begin{abstract}
We investigate the van der Waals or interaction energy due to the plasmon modes in bilayer fermion and boson systems for several layer separation and coupling strength values. Interaction effects are studied within the random-phase approximation (RPA), the quasilocalized charge approximation (QLCA), and the Singwi, Tosi, Land, and Sjölander (STLS) models of the dielectric function formalism. We find that the interaction becomes repulsive at short separation distances for strongly coupled systems described by the QLCA and STLS approaches in contrast to attractive behavior predicted by the RPA. At larger separation distances, the interaction energy changes sign within the QLCA and STLS models leading to an attractive interaction. The evident relation between our calculations and the Casimir effect is emphasized.
\end{abstract}

DOI: $10.1103 /$ PhysRevB.81.115326

PACS number(s): 73.20.Mf, 42.50.Pq, 52.27.Gr, 73.21.Ac

\section{INTRODUCTION}

The intriguing macroscopic attractive force due to the vacuum fluctuations between two parallel conductors at small separations, or the Casimir force, has been known more than half a century since Casimir published his seminal papers. ${ }^{1}$ Importance of the Casimir effect in technology is evident today. With the advanced technology, it is now routine to fabricate micro- and nanoelectromechanical systems (MEMS and NEMS), and many-layer quantum well structures, which have many applications in engineering and basic science. Observations revealed that the vacuum and charge fluctuations play a fundamental role in the performance of MEMS and NEMS. Components of these devices attract each other and stick to one another due to the Casimir effect. Hence, the Casimir effect and its dependence on material parameters, geometry, topology, and temperature in submicron systems have been a subject of intense research in experimental and theoretical condensed matter physics. ${ }^{2}$ It has also generated substantial interest in many research fields such as quantum chromodynamics, biology, astrophysics, cosmology, and mathematical physics. ${ }^{3}$

Even more intriguing aspect of the Casimir force is that it can become repulsive when real materials, i.e., dielectrics and normal conductors, are arranged in a certain way determined by their dielectric permittivities. ${ }^{4}$ A repulsive Casimir force results in nanolevitation, which may lead to ultralowfriction device technology. A recent experimental study has shown that when the optical properties of the materials are properly chosen, the long-range QED forces between solid bodies can become repulsive. ${ }^{5}$ Feiler et $a l^{6}$ measured a repulsive van der Waals (vdW) force between a gold sphere and polytetrafluoroethylene surface in cyclohexane. Indeed, they could tune the force from repulsive to attractive by changing the refractive index of the liquid. There are also some theoretical studies ${ }^{7}$ which report that the metamaterials are good candidates for the realization of repulsive Casimir force.

At short distances interaction energy due to the coupling between the surface plasmons of the bodies is the familiar $\mathrm{vdW}$ energy. ${ }^{8,9}$ The full retarded vdW energy, however, is usually called as the Casimir energy, and it has contributions from photonic and plasmonic modes in real material systems. Although current experiments can only measure their sum, investigating these modes separately might provide us information about the sign and strength of the Casimir force. In computing this force at distances large compared to $\tilde{c} / \omega$, with $\widetilde{c}$ as the speed of light in the medium, one should take into account the retardation effects as a consequence of the finite speed of light. At these distances, photon interactions dominate the Coulomb interaction.

In the present work, we will explore the contribution associated with the plasmon modes to the Casimir energy. The plasmonic contribution dominates the Casimir effect at short distances, i.e., $d \ll \lambda_{p}$. Here $d$ is the mirror separation and $\lambda_{p}$ is the plasma wavelength associated with the metal. Importance of the plasmon modes in the Casimir effect has been elucidated extensively in Ref. 10. Sernelius and Björk ${ }^{11}$ studied the interaction energy between two quantum wells, which were treated as strictly two-dimensional (2D) metallic sheets, within the RPA model; and performed both retarded and nonretarded calculations. They distinguished three regions of separation distance where contributions of different excitations become dominant. Boström and Sernelius ${ }^{12}$ later extended this calculation to include the finite-temperature effects. Intravaia and Lambrecht ${ }^{13}$ investigated role of the surface plasmons in the Casimir force between two infinitely large plane mirrors at zero temperature, and found out that one of the plasmonic modes gives repulsive contribution. It is concluded that the surface plasmon contribution is important also at large distances. In a succeeding paper, ${ }^{14}$ the authors obtained analytical expressions for the plasmonic contribution at small and large distance asymptotics. Moreover, their calculations yield a sign change, or a crossover from attractive to repulsive, for the plasmonic contribution at short distances $\left(d \approx 0.08 \lambda_{p}\right)$. Lau et al. studied the zero-point fluctuations of the plasmon modes of bilayer Wigner crystals at zero and finite temperatures. ${ }^{15}$

Barton calculated the Casimir energy of spherical plasma shells inspired by carbon molecule $\mathrm{C}_{60}$ by considering a standard hydrodynamic plasma model. ${ }^{16} \mathrm{He}$ then investigated the cohesive Casimir energies for a thin flat sheet at zero temperature by calculating the contributions from the surface plasmons and photons. ${ }^{17}$ In addition the nonretarded, per- 
fectly reflecting and the no-cutoff limits were discussed. He also studied the effects determined directly by the Maxwell fields, and calculated the self-stress of a single plasma sheet. ${ }^{18}$

Bordag et $a l .{ }^{19}$ obtained the spectrum of a flat plasma sheet model. They solved Maxwell equations with suitable boundary conditions at the plasma layer by employing Hertz potentials. Spectrum of their model consists of continuous branches as well as surface plasmons. Bordag has studied the Casimir force between two dielectric bodies and for two infinitely thin plasma sheets by investigating the contributions of surface plasmons and the photon modes. He concluded that at small distances, the plasmon contributions dominate the vacuum energy. ${ }^{20}$

Dobson et al. ${ }^{21}$ investigated the dispersion interaction in nanostructures with different geometries. They showed that the usual sum of $R^{-6}$ contributions from elements separated by distance $R$ may give qualitatively wrong results for the nonretarded vdW interaction. Recently, by employing a coupled-plasmon approach and using electron hydrodynamics, White and Dobson ${ }^{22}$ have studied the dispersion interaction between two collinear quasi-one-dimensional structures separated by a vacuum gap in the nonretarded regime. They have found that the coupled plasmon energy continues to grow with the wire length at fixed separation.

Plasmons are the well-defined collective excitations of a charged system when its dielectric function (DF) vanishes, i.e., $\varepsilon(\mathbf{q}, \omega)=0$. In an uncoupled bilayer system, each layer has a 2 D plasma mode with a long-wavelength $(q \rightarrow \infty)$ dispersion $\omega(\mathbf{q}) \sim \sqrt{q}$, where $q \equiv|\mathbf{q}|, \mathbf{q}=\left(q_{x}, q_{y}\right)$ is the 2D wave vector. As the layers get closer, the interlayer Coulomb interaction gets stronger and the system displays two different longitudinal modes: in-phase (optic) mode with energy $\hbar \omega_{+}(\mathbf{q})$ in which the charge density oscillations in the layers are in unison, and out-of-phase (acoustic) mode with energy $\hbar \omega_{-}(\mathbf{q})$, where the oscillation phase of the layers differs by $180^{\circ}$. Both modes tend to the single-layer plasmon mode $\hbar \omega_{p}(\mathbf{q})$ as the layer separation $d \rightarrow \infty$. Thus, by subtracting energy of the single-layer modes we can find the interaction energy due to the correlations between the surface plasmons in different layers as

$$
\begin{aligned}
E(d)= & \sum_{\mathbf{q}}\left[\frac{\hbar \omega_{+}(\mathbf{q})}{2}+\frac{\hbar \omega_{-}(\mathbf{q})}{2}\right]_{d \rightarrow \infty}^{d}=\frac{\hbar}{2} \sum_{\mathbf{q}}\left[\omega_{+}(\mathbf{q}, d)\right. \\
& \left.+\omega_{-}(\mathbf{q}, d)-2 \omega_{p}(\mathbf{q})\right] .
\end{aligned}
$$

This energy is considered to be the plasmonic contribution to the vdW energy or the nonretarded Casimir energy. For a method to calculate the plasmon dispersions including the retardation effects see, for instance, Refs. 10 and 23.

The present work is devoted to the computation of plasmonic contributions to the Casimir energy in strongly coupled bilayer fermion and boson systems at zero temperature within the DF formalism. We are motivated by the previous calculations ${ }^{11,13,14}$ emphasizing the role of plasmons but were confined mainly to the RPA.

In strongly coupled bilayer systems correlation effects beyond the RPA need to be taken into account. Making use of available quantum Monte Carlo (QMC) simulation data we construct models for the DF from which the plasmon dispersions are obtained. Through numerical computations we find that such correlations give rise to a sign change in the plasmonic contribution to the Casimir energy.

In the following we briefly describe calculation of the plasmon dispersions in electron and charged-boson (CB) bilayers within various DF models. In Sec. III, we present our numerical results for the plasmonic and photonic contributions to the Casimir energy.

\section{MODEL AND THEORY}

We envisage two layers of charged particles (electrons or bosons) with equal density $n$ separated by a distance $d$, which is comparable to the Wigner-Seitz radius in a singlelayer $a=1 / \sqrt{\pi n}$. Our system is thus a symmetric bilayer. Each layer is assumed to be embedded in a rigid uniform background of opposite charge so that the whole system is neutral. Furthermore, finite thickness effects, disorder effects, and interlayer tunneling are ignored.

Each layer is characterized by the dimensionless coupling parameter $r_{s}=a / a_{B}^{*}$, where $a_{B}^{*}=\hbar^{2} \epsilon /\left(e^{2} m^{*}\right)$ is the effective Bohr radius in terms of dielectric constant of the background medium $\epsilon=\kappa \epsilon_{0}$, and band effective mass of the particle $m^{*}$. The charged particles in layers 1 and 2 interact via Coulomb potentials (in Fourier space) $v_{11}(q)=v_{22}(q)=v_{c}(q)$ $=2 \pi e^{2} /(\epsilon q)$, and $v_{12}(q)=v_{21}(q)=v_{c}(q) e^{-q d}$. The energies in the subsequent discussion are presented in effective Rydberg, $R y^{*}=\hbar^{2} /\left(2 m^{*} a_{B}^{* 2}\right)=\left(r_{s}^{2} / 2\right) E_{F}$, units to make comparison between the electron and CB bilayers possible.

For symmetric bilayers the DF matrix can be diagonalized by separating the in-phase $(+)$ and out-of-phase $(-)$ directions. The matrix elements $\varepsilon_{ \pm}(\mathbf{q}, \omega)=\varepsilon_{11}(\mathbf{q}, \omega) \pm \varepsilon_{12}(\mathbf{q}, \omega)$ are obtained in terms of $v_{ \pm}(q)=v_{11}(q) \pm v_{12}(q)$, and the Lindhard function $\chi_{0}(\mathbf{q}, \omega)$ as

$$
\varepsilon_{ \pm}(\mathbf{q}, \omega)=1-\frac{v_{ \pm}(q) \chi_{0}(\mathbf{q}, \omega)}{1+v_{c}(q) \chi_{0}(\mathbf{q}, \omega)\left[G_{11}(\mathbf{q}) \pm G_{12}(\mathbf{q})\right]} .
$$

Short-range exchange and Coulomb correlation effects among the particles are taken into account in the DF through intra- and interlayer static local-field (LF) factors, i.e., $G_{11}(\mathbf{q})$ and $G_{12}(\mathbf{q})$, respectively. Different assumptions in the formulation of the DF give rise to distinct forms for the LF factors.

The calculations in this study are based on three different approaches within the DF formalism: random-phase approximation (RPA), quasilocalized charge approximation ${ }^{24}$ (QLCA), and self-consistent field theory of Singwi, Tosi, Land, and Sjölander ${ }^{25}$ (STLS). While the RPA is quite well in describing the physical properties of high-density (low $r_{s}$ ) systems, the STLS approach is more successful for the lowdensity systems. The QLCA, on the other hand, has been developed to satisfy the third-frequency-moment $\left(\left\langle\omega^{3}\right\rangle\right)$ sum rule, which is necessary for the correct calculation of the plasmon dispersion in the long-wavelength limit. It has been shown that the QLCA predicts a correlation-induced energy gap $\omega_{-}(q \rightarrow 0)$ in both electron ${ }^{26}$ and $\mathrm{CB}^{27}$ bilayer systems.

In terms of the intra- and interlayer static structure factors $S_{i j}(\mathbf{q})$, the LF factors in the QLCA read 


$$
\begin{aligned}
& G_{11}(\mathbf{q})=-\frac{1}{N} \sum_{\mathbf{k}} \frac{(\mathbf{q} \cdot \mathbf{k})^{2}}{q^{4}} \frac{v_{11}(k)}{v_{c}(q)}\left[S_{11}(\mathbf{q}-\mathbf{k})-S_{11}(\mathbf{k})\right] \\
&+\frac{1}{N} \sum_{\mathbf{k}} \frac{(\mathbf{q} \cdot \mathbf{k})^{2}}{q^{4}} \frac{v_{12}(k)}{v_{c}(q)} S_{12}(\mathbf{k}), \\
& G_{12}(\mathbf{q})=-\frac{1}{N} \sum_{\mathbf{k}} \frac{(\mathbf{q} \cdot \mathbf{k})^{2}}{q^{4}} \frac{v_{12}(k)}{v_{c}(q)} S_{12}(\mathbf{q}-\mathbf{k}) .
\end{aligned}
$$

On the other hand, the corresponding expressions within the STLS approximation are

$$
\begin{gathered}
G_{11}(\mathbf{q})=-\frac{1}{N} \sum_{\mathbf{k}} \frac{\mathbf{q} \cdot \mathbf{k}}{q^{2}} \frac{v_{11}(k)}{v_{c}(q)}\left[S_{11}(\mathbf{q}-\mathbf{k})-1\right], \\
G_{12}(\mathbf{q})=-\frac{1}{N} \sum_{\mathbf{k}} \frac{\mathbf{q} \cdot \mathbf{k}}{q^{2}} \frac{v_{12}(k)}{v_{12}(q)} S_{12}(\mathbf{q}-\mathbf{k}) .
\end{gathered}
$$

In the RPA, the $G_{i j}(\mathbf{q})=0$. Therefore, the RPA simplifies Eq. (2) to $\varepsilon_{ \pm}(\mathbf{q}, \omega)=1-v_{c}(q)\left[1 \pm e^{-q d}\right] \chi_{0}(\mathbf{q}, \omega)$. Note that the $S_{i j}(\mathbf{q})$ are related to the pair distribution functions $g_{i j}(\mathbf{r})$ via

$$
g_{i j}(\mathbf{r})=1+\frac{1}{N} \sum_{\mathbf{q}}\left[S_{i j}(\mathbf{q})-\delta_{i j}\right] e^{i \mathbf{q} \cdot \mathbf{r}} .
$$

\section{RESULTS AND DISCUSSION}

\section{A. Bilayer Fermion System}

As a bilayer fermion system, we choose two disorder-free electron quantum wells spaced by a distance $d$. For a noninteracting $2 \mathrm{D}$ electron gas system, the Lindhard function at zero temperature is given by ${ }^{28}$

$$
\begin{gathered}
\chi_{0}(\mathbf{q}, \omega)=-\frac{m^{*}}{\pi \hbar^{2}}\left[1-\frac{1}{2 q_{n}^{2}}\left(\sqrt{T_{+}}-\sqrt{T_{-}}\right)\right], \\
T_{ \pm} \equiv\left(\omega_{n} \pm q_{n}^{2}\right)^{2}-4 q_{n}^{2}, \omega_{n} \equiv \hbar \omega / E_{F}, q_{n} \equiv q / k_{F} .
\end{gathered}
$$

Using this relation in Eq. (2), one can numerically compute spectrum of the collective excitations in a bilayer electron system.

In order to simulate the system better, we computed the LF factors by making use of the $g_{i j}(\mathbf{r})$ generated by the QMC studies reported in Ref. 29. The plasmon dispersions computed within the QLCA for a system of two quantum wells with layer spacing $d=0.5 a$ are plotted in Fig. 1, as an example. In this figure, black dotted line is the upper bound of single-particle continuum, where the plasma modes decay into single electron-hole pairs.

We observe an energy gap of $3.049 E_{F}$ in the $\omega_{-}(\mathbf{q})$ mode. For a given density (or $r_{s}$ ), this gap decreases sharply as the layer spacing increases, and almost vanishes for $d>1.5 a$. Furthermore, it is larger in high-density systems for a given $d$ [see Fig. 11 of Ref. 26].

Because each plasma mode enters the single-particle continuum at a different (critical) $q$ value, and gets damped there, we modified the interaction energy expression [Eq. (1)] for electron bilayers as

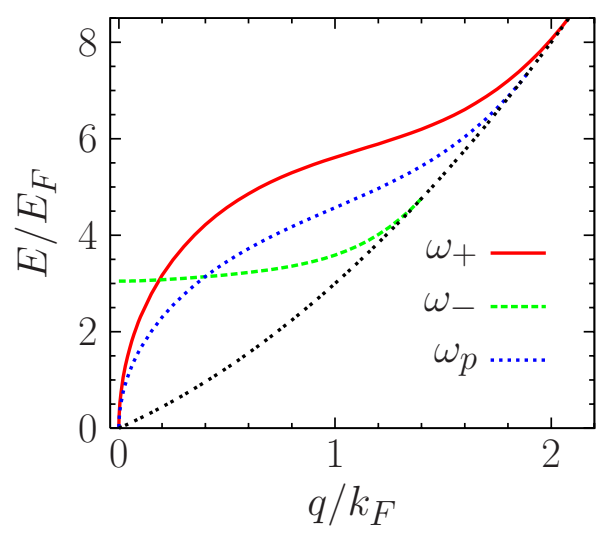

FIG. 1. (Color online) Dispersion of collective modes in a symmetric bilayer electron system with $r_{s}=10$ and $d=0.5 a$.

$$
\begin{aligned}
\frac{E(d)}{N}= & \frac{\hbar}{2} \int_{0}^{\infty} q_{n} \mathrm{~d} q_{n}\left[\omega_{+}\left(q_{n}, d\right) \Theta\left(q_{c}^{+}-q_{n}\right)\right. \\
& \left.+\omega_{-}\left(q_{n}, d\right) \Theta\left(q_{c}^{-}-q_{n}\right)-2 \omega_{p}\left(q_{n}\right) \Theta\left(q_{c}^{p}-q_{n}\right)\right] .
\end{aligned}
$$

Our method differs from that of Sernelius and Björk in the sense that they let the modes stay on the continuum boundary. We should also stress that their system was a highdensity one corresponding to $r_{s}=1.41$. In contrast, here, we consider systems with $r_{s}=10$ and 20 , where the correlations are much stronger. At these large values of $r_{s}$, we have checked numerically that both methods yield essentially the same result.

For the sake of generality, we compute the interaction energy per particle. We first present the results obtained within the RPA in Fig. 2. It is observed that the energy is negative for all layer spacings studied. The curves qualitatively show the same behavior for $0.3 \leq d / a \leq 1.5$. We found that in this interval, the interaction energy varies approximately as $d^{-5 / 2}$ at $r_{s}=10$, and $d^{-7 / 2}$ at $r_{s}=20$. This means that the power law is density dependent. Evidently, in the singlelayer limit $(d \rightarrow \infty)$ the energy goes to zero. We can obtain the force by taking the derivative of the energy with respect to the layer spacing. This procedure yields a repulsive force,

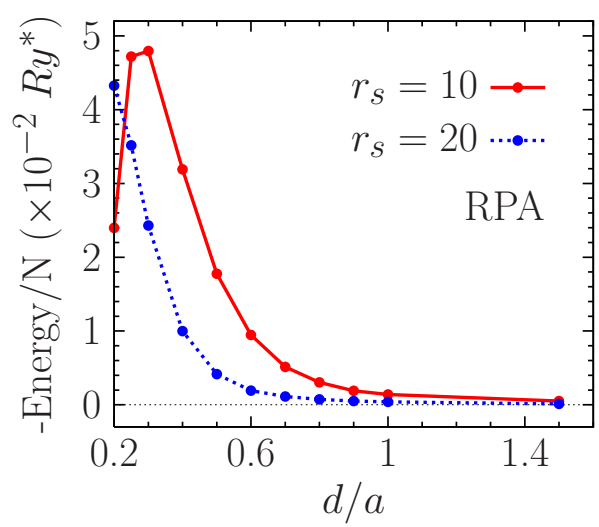

FIG. 2. (Color online) Interaction energy per particle as a function of layer spacing in an electron bilayer computed within the RPA. 


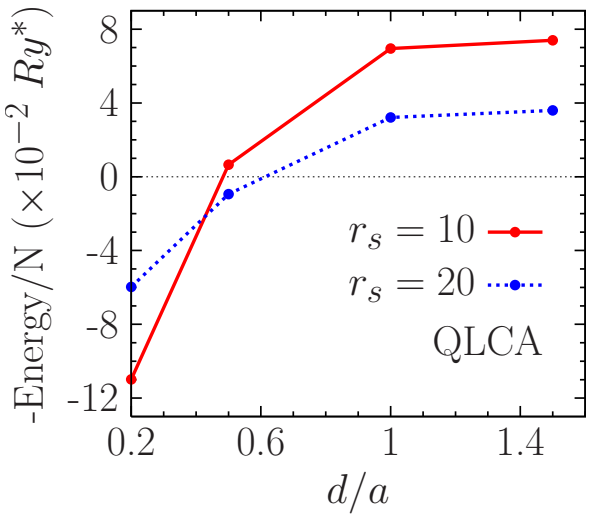

FIG. 3. (Color online) Interaction energy per particle as a function of layer separation in an electron bilayer computed within the QLCA.

which varies as $d^{-7 / 2}$ and $d^{-9 / 2}$ at respective $r_{s}$ values.

When the gap between the layers is $\sim 1 \mathrm{~cm}$, the interaction energy per particle drops to $-6.27 \times 10^{-9} R y^{*}$ and $-3.61 \times 10^{-12} R y^{*}$, respectively, when $r_{s}=10$ and 20 . Inclusion of the retardation effects results in $-3.84 \times 10^{-10} R y^{*}$ and $-2.8 \times 10^{-13} R y^{*}$ for the same $r_{s}$ values. Therefore, the retardation effects are weak for the distances $(\approx \mu \mathrm{m})$ we are interested.

The correlation-induced energy gap or more generally the correlation effects lead to quite interesting behavior in $d$ dependence in the QLCA model. Figure 3 shows that the interaction energy is positive for small $d$ values, in contrast to the RPA results, and becomes negative starting at $d=0.48 a$ for $r_{s}=10$, and $d=0.58 a$ for $r_{s}=20$. Unfortunately we have only four data points due to the available QMC data we used as input in our calculations. But it is obvious that there is a sign change in the interaction energy. This sign change shows importance of the exchange and correlation effects beyond the RPA.

We might consider two GaAs-based quantum wells as a potential experimental system. Assuming $\kappa=12.6$ and $m^{*}$ $=0.067 m_{e}$ in the conduction band, one obtains $a_{B}^{*}=99.5 \AA$. As a result, $r_{s}=10$ and 20 correspond to electron densities of $3.2 \times 10^{9} \mathrm{~cm}^{-2}$ and $8 \times 10^{8} \mathrm{~cm}^{-2}$, respectively. Also, the interparticle distance $a$ in GaAs wells is found as $\approx 100 \mathrm{~nm}$ for $r_{s}=10$, and $\approx 200 \mathrm{~nm}$ for $r_{s}=20$. These data enable us to predict the plasmonic interaction energy, and corresponding force per unit area. For example, at $r_{s}=10$ and $d=500 \AA$, we find the energy per unit area as $-1.05 \times 10^{-6} \mathrm{erg} / \mathrm{cm}^{2}$ in the RPA, and $0.38 \times 10^{-6} \mathrm{erg} / \mathrm{cm}^{2}$ in the QLCA.

In GaAs quantum wells, the envelope functions are at least $10 \mathrm{~nm}$ in width. ${ }^{30}$ This fact implies that the layer spacing typically should be $d \geq 30 \mathrm{~nm}$ to prevent tunneling between the wells. In a system with $r_{s}=10$, the spacing $d$ $=0.2 a$ corresponds to $20 \mathrm{~nm}$. Then, we conclude that our results at $d=0.2 a$ may be only qualitative for GaAs-based bilayer systems.

Photonic contribution to the interaction energy results from independent coupling of the $p$ - and $s$-polarized photons with the electric fields in the quantum wells. The energy per particle from the photon interaction may be computed through $^{11}$

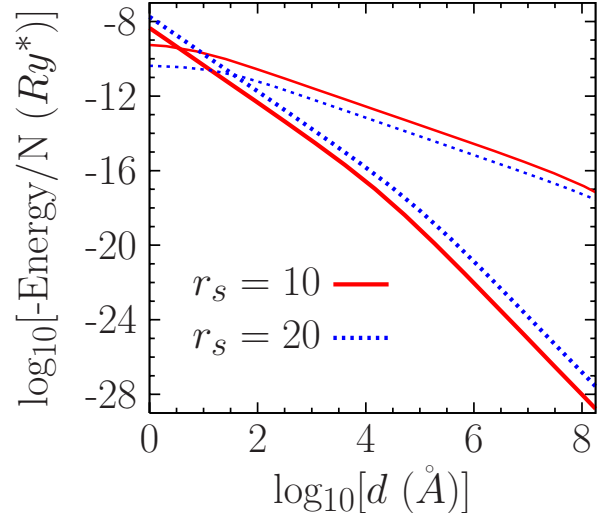

FIG. 4. (Color online) Energy contribution from the longitudinal plus $p$-polarized and $s$-polarized interactions, respectively, shown with thick and thin lines, as a function of layer separation in an electron bilayer.

$$
\frac{E_{p, s}(d)}{N}=\frac{\hbar}{4 \pi^{2} n} \int_{0}^{\infty} \int_{0}^{\infty} \mathrm{d} \omega \mathrm{d} q q \ln \left[1-e^{-2 q d \gamma(q, i \omega)} \beta_{p, s}^{2}\right] .
$$

Here $\gamma(q, i \omega)=\sqrt{1+(\omega / \widetilde{c} q)^{2}}$ is the retardation factor. In GaAs, we can assume $\widetilde{c}=c / \sqrt{\kappa}$. For the coupling between the $p$-polarized photons and longitudinal electric field of the quantum well,

$$
\beta_{p}=\frac{\gamma(q, i \omega) \alpha_{0}(q, i \omega)}{1+\gamma(q, i \omega) \alpha_{0}(q, i \omega)},
$$

where $\alpha_{0}(q, i \omega)=-v_{c}(q) \chi_{0}(q, i \omega)$ in the RPA. On the other hand, the $s$-polarized photons induce a transverse current in the well. For this interaction,

$$
\beta_{s}=\frac{2 \pi e^{2} n / q m^{*} c^{2} \gamma(q, i \omega)}{1+2 \pi e^{2} n / q m^{*} c^{2} \gamma(q, i \omega)} .
$$

Comparing with Eq. (9), we can define $2 \pi e^{2} n /\left(q m^{*} c^{2}\right)$ as the transverse polarizability.

Our results for the photonic contribution are displayed in Fig. 4. As is seen, this term is also particle density dependent similar to the plasmonic contribution, but negative for all layer spacings. It is, however, much smaller than the plasmonic contribution even up to $d=1 \mathrm{~cm}$. The $s$-photon contribution is the dominant term. We note that the $s$ - and $p$-polarization effects are calculated within the RPA model of the DF formalism.

\section{B. Bilayer Charged-Boson System}

The Casimir effect in condensed bosonic systems confined to a parallel plate geometry has been studied in detail. For example, Biswas ${ }^{31}$ investigated temperature dependence of the Casimir force for an ideal Bose gas confined between two slabs, and using the Dirichlet boundary conditions, showed that below the condensation temperature $T_{c}$ the force decreases with temperature, but independent of temperature for $T \geq T_{c}$. Also, his calculations yield a slab separation dependence for the Casimir force as $d^{-3}$ for all temperatures. 


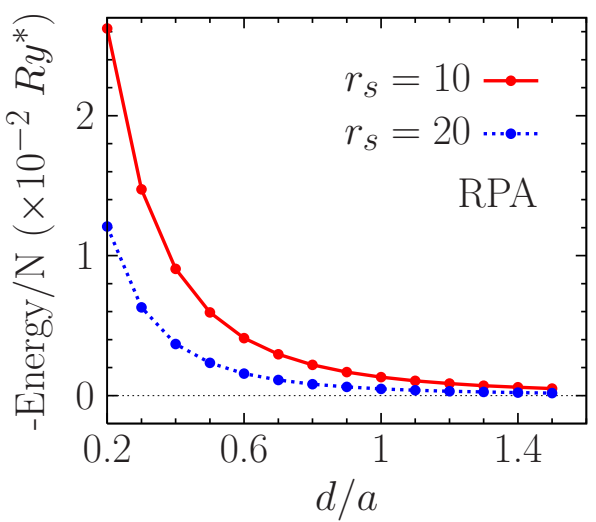

FIG. 5. (Color online) Interaction energy per particle as a function of layer spacing computed within the RPA for a CB bilayer system.

Bera and Bhattacharjee, ${ }^{32}$ however, predicted a crossover from a power law to an exponential fall off for the force depending on the size and density of the ideal Bose gas. Schiefele and $\mathrm{Henkel}^{33}$ derived a renormalized expression for the phononic Casimir energy density of a weakly interacting condensate at zero temperature in the same geometry with periodic boundary conditions.

We consider an infinite, strongly interacting CB bilayer system confined to slab geometry. The system is assumed to be in the condensed phase at zero temperature.

The response function for the noninteracting $\mathrm{CB}$ gas at zero temperature is $\chi_{0}(\mathbf{q}, \omega)=2 n E_{q} /\left[(\hbar \omega+i \eta)^{2}-E_{q}^{2}\right]$, where $E_{q}=\hbar^{2} q^{2} /\left(2 m^{*}\right)$ and $\eta$ is a positive infinitesimal quantity. Using this expression in the equation $\varepsilon(\mathbf{q}, \omega)=0$, one can easily obtain dispersion relations for the collective modes of a bilayer $\mathrm{CB}$ system as

$$
\left[\hbar \omega_{ \pm}(\mathbf{q})\right]^{2}=\frac{q_{n}^{4}}{r_{s}^{4}}+\frac{8 q_{n}}{r_{s}^{3}}\left\{1 \pm e^{-q d}-\left[G_{11}(\mathbf{q}) \pm G_{12}(\mathbf{q})\right]\right\},
$$

where $q_{n} \equiv q a$. Note that these relations are exact and analytical due to the simple form of the $\chi_{0}(\mathbf{q}, \omega)$. The RPA results are obtained again by setting $G_{i j}(\mathbf{q})=0$. We refer the reader to our previous work ${ }^{27}$ for the excitation spectrum and some other physical properties of CB bilayers for a wide range of system parameters.

The RPA interaction energy results are depicted in Fig. 5. They display the same qualitative behavior as the electron bilayer system. We find that the energy varies with spacing as $d^{-1.91}$ for $r_{s}=10$, and $d^{-2.05}$ for $r_{s}=20$ in the range 0.3 $\leq d / a \leq 1.5$. Hence, the force is repulsive, and varies approximately as $d^{-3}$.

In our investigations within the QLCA and STLS, we resorted to the $S(\mathbf{q})$ data obtained via reptation QMC method by De Palo et al. ${ }^{34}$ for a single-layer CB system. On the other hand, since neither $g_{12}(\mathbf{r})$ nor $S_{12}(\mathbf{q})$ are available from the QMC simulations, we confined our calculations to approximate $S_{12}(\mathbf{q})$ within the RPA.

In the QLCA model, the energy is positive until $d$ $=1.45 a$ at $r_{s}=10$, and $d=1.4 a$ at $r_{s}=20$ values; then it be-
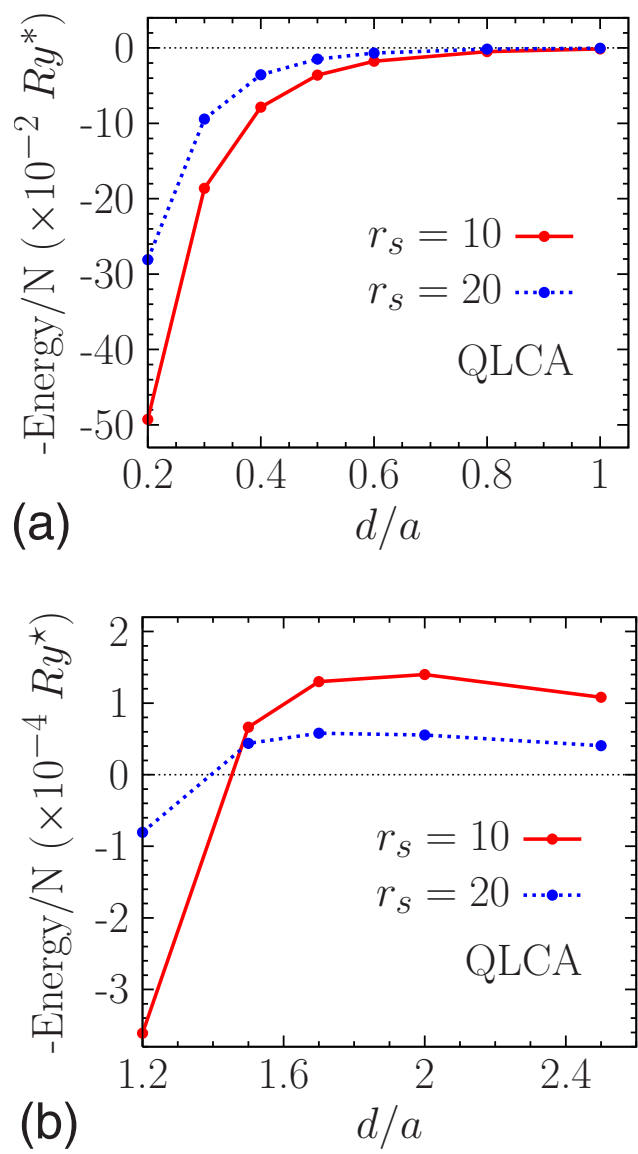

FIG. 6. (Color online) Interaction energy per particle as a function of layer spacing computed within the QLCA for a CB bilayer system.

comes negative (see Fig. 6). It varies with the layer spacing as $d^{-3.2}$, and $d^{-3.6}$ for densities, respectively, $r_{s}=10$ and 20 in the range $0.3 \leq d / a \leq 1.5$. Thus, the attractive/repulsive nature of the Casimir force due to plasmonic contributions appear not to depend on charged particle statistics. The effect, however, is much smaller for charged bosons as revealed by the scales of Figs. 3 and 6(b). The main reason for this difference in magnitudes is the fact that in the charged-boson case there are larger cancellation effects in Eq. (1) because of the greater range of $q$ values in the dispersion relations, whereas in the fermion case particle-hole continuum limits the integration [see Eq. (7)].

Notice that the energy displays a somewhat different qualitative behavior in the STLS approach than that in the QLCA. We observe two well-separated regions in Fig. 7. For small spacings it is positive but decreases and becomes negative. Then, after a minimum, it starts to increase. These differences are related to the way exchange-correlation effects are treated in different DF approaches. Hence, the sign change in the interaction energy as a function of layer separation seems to be result of beyond-RPA correlations.

We have also computed the photonic contributions to the interaction energy for the $\mathrm{CB}$ bilayers, and found similar results obtained for the electron bilayers.

In summary, we have investigated plasmonic contribution to the Casimir energy in strongly correlated fermion and 


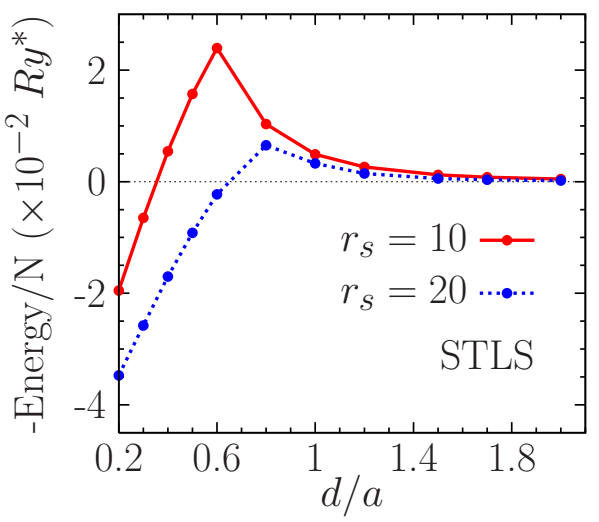

FIG. 7. (Color online) Interaction energy per particle as a function of layer spacing computed within the STLS for a CB bilayer system.

charged-boson bilayer systems confined to parallel plate geometry at zero temperature. Our analysis is based on different models of the dielectric formalism. Our findings explicitly show the importance of intra- and interlayer Coulomb interactions. Inclusion of the interactions beyond the RPA via local-field corrections gives rise to sign change in the plas- monic interaction energy and force. Plasmonic contributions to the nonretarded Casimir energy are dominant for layer spacings available in typical devices. We have also obtained some power laws for the layer spacing dependence of the energy. Variation in the Casimir energy with interlayer distance is particle density dependent. Indeed, this observation has been experimentally verified recently by Chen et al. ${ }^{35}$ Their measurement of the Casimir force between a gold coated sphere and two silicon plates of different particle densities using atomic force microscopy revealed that the $\mathrm{Ca}$ simir interaction can be modified by changing the carrier density of the semiconductor plate by several orders of magnitude. It would be most interesting to perform Casimir force experiments in the strongly interacting regime.

\section{ACKNOWLEDGMENTS}

The authors are thankful to G. Senatore and S. Moroni for sending them the QMC data, respectively, for the pair distribution function of a bilayer electron system, and the static structure function of a single-layer charged-boson system. This work is supported by TUBITAK (Grant No. 108T743) and TUBA.
${ }^{1}$ H. B. G. Casimir, Proc. K. Ned. Akad. Wet. 51, 793 (1948); H. B. G. Casimir and D. Polder, Phys. Rev. 73, 360 (1948); H. B. G. Casimir, J. Chim. Phys. Phys.-Chim. Biol. 46, 407 (1949).

${ }^{2}$ M. Bordag, U. Mohideen, and V. M. Mostepanenko, Phys. Rep. 353, 1 (2001), and references therein; H. B. Chan, V. A. Aksyuk, R. N. Kleiman, D. J. Bishop, and F. Capasso, Science 291, 1941 (2001); Phys. Rev. Lett. 87, 211801 (2001); E. Buks and M. L. Roukes, Phys. Rev. B 63, 033402 (2001); C. Genet, A. Lambrecht, and S. Reynaud, Eur. Phys. J. Spec. Top. 160, 183 (2008); A. Katzenmeyer, V. P. Logeeswaran, B. Tekin, and M. S. Islam, Proceedings of the 2nd IEEE International Nanoelectronics Conference, INEC (2008), p. 166.

${ }^{3}$ G. Plunien, B. Müller, and W. Greiner, Phys. Rep. 134, 87 (1986); E. Elizalde and A. Romeo, Am. J. Phys. 59, 711 (1991); F. Belgiorno and S. Liberati, Gen. Relativ. Gravit. 29, 1181 (1997); P. A. Pincus and S. A. Safran, Europhys. Lett. 42, 103 (1998); M. Kardar and R. Golestanian, Rev. Mod. Phys. 71, 1233 (1999); K. A. Milton, The Casimir Effect: Physical Manisfestations of Zero-Point Energy (World Scientific, Singapore, 2001); Y. Levin, Rep. Prog. Phys. 65, 1577 (2002); S. K. Lamoreaux, ibid. 68, 201 (2005); H. Ahmedov and I. H. Duru, J. Math. Phys. 46, 022303 (2005).

${ }^{4}$ E. M. Lifshitz, Sov. Phys. JETP 2, 73 (1956); I. E. Dzyaloshinskii, E. M. Lifshitz, and L. P. Pitaevskii, Sov. Phys. Usp. 73, 381 (1961); Jacob N. Israelachvili, Intermolecular and Surface Forces, (Academic Press, London, 1992); V. Adrian Parsegian, Van der Waals Forces (Cambridge University Press, New York, 2006).

${ }^{5}$ J. N. Munday, F. Capasso, and V. A. Parsegian, Nature (London) 457, 170 (2009).

${ }^{6}$ A. A. Feiler, L. Bergström, and M. W. Rutland, Langmuir 24, 2274 (2008).

${ }^{7}$ R. Zhao, J. Zhou, T. Koschny, E. N. Economou, and C. M.
Soukoulis, Phys. Rev. Lett. 103, 103602 (2009) and references therein.

${ }^{8}$ N. G. Van Kampen, B. R. A. Nijboer, and K. Schram, Phys. Lett. A 26, 307 (1968)

${ }^{9}$ E. Gerlach, Phys. Rev. B 4, 393 (1971).

${ }^{10}$ Bo E. Sernelius, Surface Modes in Physics (Wiley-VCH, Mörlenbach, 2001).

${ }^{11}$ Bo E. Sernelius and P. Björk, Phys. Rev. B 57, 6592 (1998).

${ }^{12} \mathrm{M}$. Boström and Bo E. Sernelius, Microelectron. Eng. 51-52, 287 (2000).

${ }^{13}$ F. Intravaia and A. Lambrecht, Phys. Rev. Lett. 94, 110404 (2005).

${ }^{14}$ F. Intravaia, C. Henkel, and A. Lambrecht, Phys. Rev. A 76, 033820 (2007).

${ }^{15}$ A. W. C. Lau, D. Levine, and P. Pincus, Phys. Rev. Lett. 84, 4116 (2000); A. W. C. Lau, P. Pincus, D. Levine, and H. A. Fertig, Phys. Rev. E 63, 051604 (2001).

${ }^{16}$ G. Barton, J. Phys. A 37, 1011 (2004).

${ }^{17}$ G. Barton, J. Phys. A 38, 2997 (2005) and references therein.

${ }^{18}$ G. Barton, J. Phys. A 38, 3021 (2005).

${ }^{19}$ M. Bordag, I. G. Pirozhenko, and V. V. Nesterenko, J. Phys. A 38, 11027 (2005).

${ }^{20}$ M. Bordag, J. Phys. A 39, 6173 (2006).

${ }^{21}$ J. F. Dobson, A. White, and A. Rubio, Phys. Rev. Lett. 96, 073201 (2006)

${ }^{22}$ A. White and J. F. Dobson, Phys. Rev. B 77, 075436 (2008).

${ }^{23}$ H. Raether, Surface Plasmons on Smooth and Rough Surfaces and on Gratings (Springer, Berlin, 1988).

${ }^{24}$ G. Kalman and K. I. Golden, Phys. Rev. A 41, 5516 (1990); H. Mahassen, Ph.D. thesis, The University of Vermont, 2007.

${ }^{25}$ K. S. Singwi, M. P. Tosi, R. H. Land, and A. Sjölander, Phys. Rev. 176, 589 (1968).

${ }^{26}$ K. I. Golden, H. Mahassen, G. J. Kalman, G. Senatore, and F. 
Rapisarda, Phys. Rev. E 71, 036401 (2005).

${ }^{27}$ M. Tas and B. Tanatar, Phys. Rev. B 78, 115324 (2008).

${ }^{28}$ S. Das Sarma, J. K. Jain, and R. Jalabert, Phys. Rev. B 41, 3561 (1990).

${ }^{29}$ B. Tanatar and D. M. Ceperley, Phys. Rev. B 39, 5005 (1989); F. Rapisarda, Ph.D. thesis, University of Trieste, 1995; F. Rapisarda and G. Senatore, in Strongly Coupled Coulomb Systems, edited by G. J. Kalman, J. M. Rommel, and K. Blagoev (Plenum Press, New York, 1998); G. Senatore (private communication).
${ }^{30}$ J. Szymański, L. Świerkowski, and D. Neilson, Phys. Rev. B 50, 11002 (1994).

${ }^{31}$ S. Biswas, Eur. Phys. J. D 42, 109 (2007).

${ }^{32}$ N. Bera and J. K. Bhattacharjee, Eur. Phys. J. D 53, 21 (2009).

${ }^{33}$ J. Schiefele and C. Henkel, J. Phys. A 42, 045401 (2009).

${ }^{34}$ S. De Palo, S. Conti, and S. Moroni, Phys. Rev. B 69, 035109 (2004).

${ }^{35}$ F. Chen, G. L. Klimchitskaya, V. M. Mostepanenko, and U. Mohideen, Phys. Rev. Lett. 97, 170402 (2006). 